\title{
RESEARCH
}

\section{Crystal structure of a ligand-free stable TSH receptor leucine-rich repeat domain}

\author{
Jennifer Miller-Gallacher, Paul Sanders, Stuart Young, Andrew Sullivan, Stuart Baker, Samuel C Reddington, \\ Matthew Clue, Katarzyna Kabelis, Jill Clark, Jane Wilmot, Daniel Thomas, Monika Chlebowska, Francesca Cole, \\ Emily Pearson, Emma Roberts, Matthew Holly, Michele Evans, Ricardo Núñez Miguel, Michael Powell, \\ Jane Sanders, Jadwiga Furmaniak and Bernard Rees Smith \\ FIRS Laboratories, RSR Ltd, Cardiff, CF14 5DUUK
}

Correspondence should be addressed to B R Smith: firs@rsrltd.eclipse.co.uk

\begin{abstract}
The crystal structures of the thyroid-stimulating hormone receptor (TSHR) leucine-rich repeat domain (amino acids 22-260; TSHR260) in complex with a stimulating human monoclonal autoantibody (M22 ${ }^{\mathrm{TM}}$ ) and in complex with a blocking human autoantibody (K1-70' ${ }^{\mathrm{TM}}$ ) have been solved. However, attempts to purify and crystallise free TSHR260, that is not bound to an autoantibody, have been unsuccessful due to the poor stability of free TSHR260. We now describe a TSHR260 mutant that has been stabilised by the introduction of six mutations (H63C, R112P, D143P, D151E, V169R and I253R) to form TSHR260-JMG55TM, which is approximately 900 times more thermostable than wild-type TSHR260. These six mutations did not affect the binding of human TSHR monoclonal autoantibodies or patient serum TSHR autoantibodies to the TSHR260. Furthermore, the response of full-length TSHR to stimulation by TSH or human TSHR monoclonal autoantibodies was not affected by the six mutations. Thermostable TSHR260-JMG55TM has been purified and crystallised without ligand and the structure solved at $2.83 \AA$ resolution. This is the first reported structure of a glycoprotein hormone receptor crystallised without ligand. The unbound TSHR260-JMG55TM structure and the M22 and K1-70 bound TSHR260 structures are remarkably similar except for small changes in side chain conformations. This suggests that neither the mutations nor the binding of $\mathrm{M} 22^{\mathrm{TM}}$ or K1-70'M change the rigid leucine-rich repeat domain structure of TSHR260. The solved TSHR260-JMG55TM structure provides a rationale as to why the six mutations have a thermostabilising effect and provides helpful guidelines for thermostabilisation strategies of other soluble protein domains.
\end{abstract}

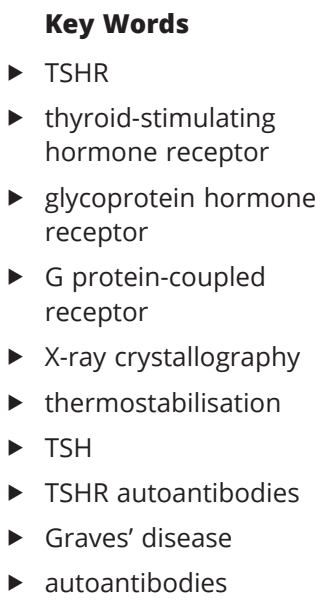

Journal of Molecular Endocrinology (2019) 62, 117-128

\section{Introduction}

The thyroid-stimulating hormone (TSH) receptor (TSHR) is involved in the control of thyroid function and is a major autoantigen in autoimmune thyroid diseases (Rees Smith et al. 1988, 2007, McKenzie \& Zakarija 1992, Sanders et al. 1997, Rapoport et al. 1998, 2015, Evans et al. 2010,
Furmaniak et al. 2013, 2015). TSHR is a member of the glycoprotein hormone receptor sub-family of the leucinerich repeat-containing family of class A G protein-coupled receptors (GPCRs; Hsu et al. 2000, Kleinau et al. 2017, Núñez Miguel et al. 2017), which includes the follicle-stimulating 
hormone receptor and the luteinising hormone receptor. The first 21 amino acids of the TSHR make up the cleaved signal peptide with the remaining mature TSHR consisting of three domains: the N-terminal extracellular leucine-rich repeat (LRR) domain (LRD, residues 22-281), the hinge region or cleavage domain (CD, residues 282-409) and the transmembrane domain (TMD, residues 410-764) with an intracellular C-terminus (Núñez Miguel et al. 2004). TSHR undergoes intramolecular cleavage at the hinge region with approximately 50 amino acids removed ( 317-366) leaving two components, subunit A which contains the LRD and subunit B which consists largely of the TMD, linked by two disulphide bonds (Rees Smith et al. 1988, Sanders et al. 1997, Chazenbalk et al. 1997a,b, Tanaka et al. 1998, de Bernard et al. 1999, Rapoport \& McLachlan 2016). TSHR autoantibodies (TRAb) with stimulating activity are responsible for hyperthyroidism in Graves' disease while TRAb with blocking activity may cause hypothyroidism. Both, stimulating- and blocking-type TRAb bind to the TSHR LRD (Rees Smith et al. 2007, 2009, Evans et al. 2010).

The crystal structures of residues 22-260 of wild-type (WT) TSHR (TSHR260; comprising most of the LRD) in complex with the antigen-binding fragments (Fab) of both a stimulating human monoclonal autoantibody (mAb), M22 ${ }^{\mathrm{TM}}$ (Sanders et al. 2003, 2007) and a blocking human mAb, K1-70 ${ }^{\mathrm{TM}}$ (Evans et al. 2010, Sanders et al. 2011) have been solved. The structures of TSHR260 bound to M22 or to $\mathrm{K} 1-70^{\mathrm{TM}}$ are remarkably similar to each other with the root mean square deviation (RMSD) of all $\mathrm{C}_{\alpha}$ atoms in the two structures being only $0.5 \AA$ (Sanders et al. 2011). However, whether the structure of a ligand-free TSHR260 changes upon the ligand binding could not be determined, as attempts to purify and crystallise free TSHR260 have not been successful to date. The principal problem is the propensity of ligand-free TSHR260 to unfold and degrade during purification (Sanders et al. 2007).

Purification and crystallisation of other unstable GPCRs, such as the $\beta_{1}$-adrenergic receptor, was accomplished by systematically mutating every residue and identifying which mutations improved thermostability (SerranoVega et al. 2008). Combined mutations improved the thermostability of the adenosine $\mathrm{A}_{2 \mathrm{~A}}$ receptor (226-fold; Lebon et al. 2011) and of the $\beta_{1}$-adrenergic receptor (900fold; Miller \& Tate 2011). Such increased thermostability has enabled the purification and crystallisation of several membrane proteins including GPCRs, transporters and ion channels (Magnani et al. 2016).

We now describe a similar approach to produce a thermostable mutant of TSHR260 (TSHR260-JMG55TM), which contains six thermostabilising mutations and shows TRAb binding activity similar to WT TSHR260. Ligand-free TSHR260-JMG55 ${ }^{\mathrm{TM}}$ was purified, crystallised and the structure solved to $2.83 \AA$ Aresolution. Comparison of the ligand-free TSHR260-JMG55'TM structure with the structures of TSHR260 in complex with M22 ${ }^{\mathrm{TM}}$ or K1-70 ${ }^{\mathrm{TM}}$ showed that the structure of TSHR260 remained essentially unchanged upon ligand binding.

\section{Materials and methods}

Rational scanning mutagenesis of TSHR260 (residues 22-260 of TSHR) coupled to a thermostability assay was used to improve the thermostability of TSHR260. Each residue of WT TSHR260 was mutated to an amino acid estimated to have the highest probability of stabilising TSHR260 for each position of TSHR260 according to sequence, structure and computational-based methods. The mutated TSHR260 were transiently expressed in CHO-K1 cells and the activity and thermostability of the mutants assessed. The most thermostabilising mutations were then combined and assessed to find increasingly more thermostable mutants that retained the ability of WT TSHR260 to bind TRAbs.

\section{Mutagenesis and expression of TSHR260 mutants}

A TSHR260-6His construct, coding amino acids $1-260$ of the human TSHR with a six-histidine tag at the C-terminus (Sanders et al. 2007) in the plasmid pcDNA3.1+ (Invitrogen) was used as a template for mutagenesis. Mutants of TSHR260 were generated by PCR-based site-directed mutagenesis using KOD Hot Start DNA Polymerase (Merck) and the plasmid pcDNA3.1-TSHR260-6His, or the appropriate TSHR260 mutants, as a template. XL1-Blue competent cells (Agilent Technologies) were transformed with PCR assays and cDNAs of individual clones fully sequenced to ascertain the presence of the desired mutations.

TSHR260 mutants were transiently transfected into CHO-K1 cells using FreeStyle MAX Reagent (Invitrogen) and expressed TSHR260 proteins secreted into the media were harvested from the wells, centrifuged at $13,000 \mathrm{rpm}$ for $2 \mathrm{~min}$ to remove the cell debris and stored at $-70^{\circ} \mathrm{C}$.

\section{TSHR260-binding assay}

TSHR260 preparations were immobilised on ELISA plate wells coated with $14 \mathrm{C} 4-\mathrm{Fab}_{2}$ (a mouse mAb that binds to a conformational epitope on the TSHR260) to measure the autoantibody binding activity of TSHR260 in a sample. The amount of active TSHR260 bound was detected using 
M22-peroxidase (M22-POD; RSR Ltd, Cardiff, UK, Rees Smith et al. 2004) (Fig. 1A). The first batch of WT TSHR260 standard expressed (stored in aliquots at $-70^{\circ} \mathrm{C}$ ) was assigned the arbitrary activity of $100 \mathrm{U} / \mathrm{mL}$ and used to determine the relative activity of mutant TSHR260 samples.

\section{Thermostability assay}

TSHR260 mutants, transiently expressed in CHO-K1 cells, were heated for between 0 and $3 \mathrm{~h}$ at $42^{\circ} \mathrm{C}, 50^{\circ} \mathrm{C}, 55^{\circ} \mathrm{C}$ or $60^{\circ} \mathrm{C}$ or for between 0 and 30 days at $37^{\circ} \mathrm{C}$. The amount of active TSHR260 in each sample was measured in the TSHR260-binding assay described above (Fig. 1B). Assay data were plotted against time and fitted to an exponential curve and the half-life $\left(t_{1 / 2}\right)$ of the mutants calculated using GraphPad Prism (GraphPad Software Inc. 2015).

\section{M22-POD affinity assay}

The TSHR260-binding assay as described above was adapted, using M22-POD at a range of concentrations. GraphPad Prism was used to plot the binding curve for M22-POD correcting for non-specific binding. Non-linear regression (one-site specific binding saturation curve) was used to calculate the equilibrium binding constant, $\mathrm{K}_{\mathrm{d}}$.

\section{Inhibition assays}

The TSHR260 binding assay described above with either WT TSHR260 or TSHR260-JMG55 bound to 14C4-coated

A

14C4-coated ELISA plate well + TSHR260 mutant
$\downarrow$ Capture
-14C4-TSHR260 mutant
$\downarrow+$ M22-POD
-14C4-TSHR260 mutant-M22-POD
$\downarrow+$ TMB
Colour change proportional to M22-POD bound to active TSHR260 mutant

B
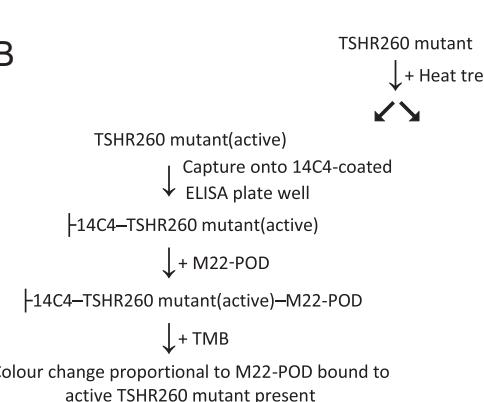

$\downarrow+$ Heat treatment
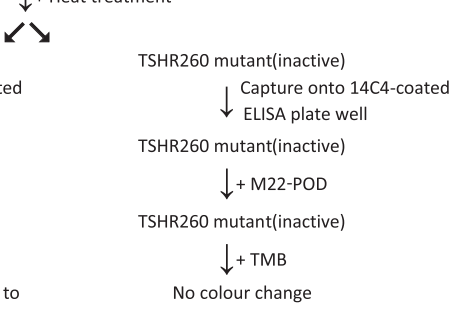

Figure 1

Schematic diagrams of (A) TSHR260-binding assay and (B) TSHR260 thermostability assay. Reproduced with permission of the copyright holder, RSR Ltd. plate wells was used to detect inhibition of M22-POD binding by either healthy blood donor sera (N1-N4) or Graves' patient sera (G1-G10).

\section{Cyclic AMP assays}

The mutations of the most thermostable TSHR260 mutant, TSHR260-JMG55TM were transferred to the full-length TSHR nucleotide sequence in pcDNA5.1/ FRT vector (Sanders et al. 2006) to produce full-length TSHR-JMG55TM. Full-length WT TSHR and full-length TSHR-JMG55TM constructs were stably transfected into CHO cells using the Flp-In system (Invitrogen) and the ability of porcine TSH and human TSHR mAbs M22 ${ }^{\mathrm{TM}}$ and $\mathrm{K} 1-18^{\mathrm{TM}}$ to stimulate production of cyclic AMP in transfected $\mathrm{CHO}$ cells was tested as described previously (Sanders et al. 2006). Cyclic AMP concentration in the lysates was assayed using Direct cAMP ELISA kits (Enzo Life Sciences, Exeter, UK). GraphPad Prism was used to fit a dose-response curve using non-linear regression and calculate the EC50 of each agonist.

\section{Expression of TSHR260-JMG55TM in insect cells}

The GP64 signal peptide (residues 1-20, amino acid sequence MVSAIVLYVLLAAAAHSAFA) replaced the native signal peptide (residues 1-21 of TSHR) to improve expression levels. Baculovirus preparations were made using the Bac-to-Bac system (Invitrogen) from GP64TSHR260-JMG55TM, with a six-histidine tag at the C-terminus, in the pFastBac1 plasmid according to the manufacturer's instructions. TSHR260-JMG55 was expressed in High-Five ${ }^{\mathrm{TM}}$ insect cells (BTI-TN-5B1-4; Gibco) as previously described (Sanders et al. 2007).

\section{Purification of TSHR260-JMG55TM}

TSHR260-JMG55 ${ }^{\mathrm{TM}}$ was purified by five rounds of column chromatography using the following: (a) cation exchange chromatography on Streamline Direct HST matrix (GE Healthcare); (b) antibody affinity chromatography using 14C4-mAb coupled to sepharose 4B (Sigma-Aldrich); (c) nickel-affinity chromatography using Ni-NTA Agarose (Qiagen); (d) deglycosylated with Endoglycosidase F3 (Sigma-Aldrich); (e) then further purified by cation exchange on a TSKgel Bioassist S HPLC Column (TOSOH Bioscience, Reading, UK) and (f) size exclusion HPLC on a Superdex 75 column (GE Healthcare). 


\section{Crystallisation of TSHR260-JMG55TM and structure determination}

Purified TSHR260-JMG55TM was concentrated to $6 \mathrm{mg} /$ $\mathrm{mL}$ in $200 \mathrm{mmol} / \mathrm{L} \mathrm{NaCl}, 25 \mathrm{mmol} / \mathrm{L}$ Tris $\mathrm{pH} 8.0$ and crystallised using the hanging drop method of vapour diffusion with a precipitant solution containing $13 \% \mathrm{w} / \mathrm{v}$ PEG 3350 and $100 \mathrm{mmol} / \mathrm{L}$ Tris $\mathrm{pH}$ 8.0.

X-ray diffraction data were collected at beamline ID30A, European Synchrotron Radiation Facility, Grenoble. Data were processed using MOSFLM (Battye et al. 2011) and AIMLESS (Evans \& Murshudov 2013). The structure of TSHR260 in complex with K1-70 ${ }^{\mathrm{TM}}$ (TSHR-K1-70, PDB code 2XWT, Sanders et al. 2011) was used for molecular replacement using PHASER (McCoy et al. 2007). The model was refined using REFMAC5 (Murshudov et al. 2011) and validated with COOT (Emsley et al. 2010).MOSFLM, AIMLESS, PHASER and REFMACS were used as part of the CCP4 suite of programs (Winn et al. 2011; www.ccp4. co.uk) Figures were prepared using PyMOL (Schrödinger, LLC 2015). Structural superimpositioning was performed with Discovery Studio 3.5 (Accelrys Software Inc. 2012).

\section{Results}

\section{Thermostabilising TSHR260}

In all, 239 single point mutants of WT TSHR260 were expressed and subjected to an initial thermostability screen by heating at $42^{\circ} \mathrm{C}$ for $30 \mathrm{~min}$ and comparing the percentage survival to that of WT TSHR260. Low activity (less than 10\% of WT TSHR260) was observed in 82 out of 239 mutants (34\%) prior to heating at $42^{\circ} \mathrm{C}$. There was an increase in thermostability at $42^{\circ} \mathrm{C}$ in the case of 65 mutants $(27 \%)$ and these mutants were further analysed in the thermostability assay. In the thermostability assay, 17 (7.1\%) mutations (P28E, L59F, T62V, H63C,

Table 1 Thermostability half-lives of TSHR260 single mutants at $42^{\circ} \mathrm{C}$.

\begin{tabular}{|c|c|c|}
\hline TSHR260 mutant & $\begin{array}{l}\text { Half-life at } \\
42^{\circ} \mathrm{C}(\mathrm{min})\end{array}$ & $\begin{array}{l}\text { Half-life ratio relative } \\
\text { to TSHR260-WT }\end{array}$ \\
\hline TSHR260-WT & $31 \pm 5$ & 1 \\
\hline $\mathrm{H} 63 \mathrm{C}$ & $70 \pm 13$ & $2.2 \pm 0.5$ \\
\hline R112P & $62 \pm 14$ & $1.8 \pm 0.4$ \\
\hline D143P & $80 \pm 12$ & $3.2 \pm 0.6$ \\
\hline D151E & $53 \pm 2$ & $2.11 \pm 0.13$ \\
\hline V169R & $56 \pm 12$ & $2.3 \pm 0.5$ \\
\hline I253R & $88 \pm 19$ & $3.1 \pm 0.4$ \\
\hline
\end{tabular}

Results are expressed as mean \pm S.D. for experiments repeated at least twice. Half-life ratios are calculated relative to the half-life of TSHR260-WT measured in the same assay (see text for experimental details).
L64Y, R112P, P142I, D143P, D151E, S166T, I167F, P168Y, V169R, N170W, T179C, I253R and R255Y) were identified that increased the thermostability of WT TSHR260 at $42^{\circ} \mathrm{C}$ by at least $60 \%$ from a half-life of $31 \pm 5 \mathrm{~min}$ to $88 \pm 19 \mathrm{~min}$ for the best single mutant, I253R. The six most thermostabilising single mutants and their half-lives at $42^{\circ} \mathrm{C}$ are listed in Table 1 . The most thermostabilising mutations were combined sequentially to ultimately produce the TSHR260 mutant, TSHR260-JMG55TM, containing the mutations H63C, R112P, D143P, D151E, V169R and I253R. The half-lives of the most thermostable single (I253R), double $(\mathrm{JMG} 22=\mathrm{D} 143 \mathrm{P}+\mathrm{I} 253 \mathrm{R})$, triple $(\mathrm{JMG} 37=\mathrm{R} 112 \mathrm{P}+\mathrm{D} 143 \mathrm{P}+\mathrm{I} 253 \mathrm{R}), \quad$ quadruple $\quad(\mathrm{JMG} 45=$ $\mathrm{R} 112 \mathrm{P}+\mathrm{D} 143 \mathrm{P}+\mathrm{D} 151 \mathrm{E}+\mathrm{I} 253 \mathrm{R})$, quintuple (JMG52 $=\mathrm{H} 63 \mathrm{C}$ $+\mathrm{R} 112 \mathrm{P}+\mathrm{D} 143 \mathrm{P}+\mathrm{D} 151 \mathrm{E}+\mathrm{I} 253 \mathrm{R})$ and hextuple (JMG55 = $\mathrm{H} 63 \mathrm{C}+\mathrm{R} 112 \mathrm{P}+\mathrm{D} 143 \mathrm{P}+\mathrm{D} 151 \mathrm{E}+\mathrm{V} 169 \mathrm{R}+\mathrm{I} 253 \mathrm{R}) \mathrm{TSHR} 260$ mutants are listed in Table 2 for thermostability assays performed at $42^{\circ} \mathrm{C}, 50^{\circ} \mathrm{C}, 55^{\circ} \mathrm{C}$ and $60^{\circ} \mathrm{C}$. The improvement in thermostability between the WT TSHR260 and TSHR260-JMG55TM was too great to measure both easily in the same assay at the same temperature. However, the ratio of the half-lives of the mutants measured at different temperatures is consistent and therefore it is possible to calculate the improvement in thermostability by multiplying the ratios of the halflives at different temperatures. By this calculation, the six thermostabilising mutations of TSHR260-JMG55TM increased the thermostability of TSHR260 approximately 900-fold (Fig. 2A and Table 2).

\section{Characteristics of TSHR260-JMG55 TM}

TSHR260-JMG55 ${ }^{\mathrm{TM}}$ had similar characteristics to the WT TSHR260 in binding and inhibition assays with porcine $\mathrm{TSH}, \mathrm{mAbs} \mathrm{M} 22^{\mathrm{TM}}, \mathrm{K} 1-70^{\mathrm{TM}}$ and $\mathrm{K} 1-18^{\mathrm{TM}}$ and TRAbpositive patient sera. In particular, TSHR260-JMG55TM bound M22-POD with similar affinity as WT TSHR260 (TSHR260 $\mathrm{K}_{\mathrm{d}}=0.10 \pm 0.04 \mu \mathrm{g} / \mathrm{mL}, \quad$ TSHR260-JMG55TM $\mathrm{K}_{\mathrm{d}}=0.16 \pm 0.03 \mu \mathrm{g} / \mathrm{mL}, n=3$; Fig. $2 \mathrm{~B}$ ). Similar results were obtained for the affinity of K1-70-POD and K1-18-POD for WT TSHR260 and TSHR260-JMG55TM (data not shown). TRAb-positive patient sera (G1-G10) inhibited the binding of M22-POD to both WT TSHR260 and TSHR260JMG55TM in a similar manner, while healthy blood donor sera (N1-N4) did not inhibit the binding of M22-POD to either WT TSHR260 or TSHR260-JMG55TM (Fig. 2C).

The stimulation of full-length WT TSHR and fulllength TSHR-JMG55TM (expressed in Flp-In CHO cells) by porcine TSH, M22 ${ }^{\mathrm{TM}}-\mathrm{Fab}$ and $\mathrm{K} 1-18^{\mathrm{TM}} \mathrm{IgG}$ was determined by measuring the amount of cyclic AMP produced using a 


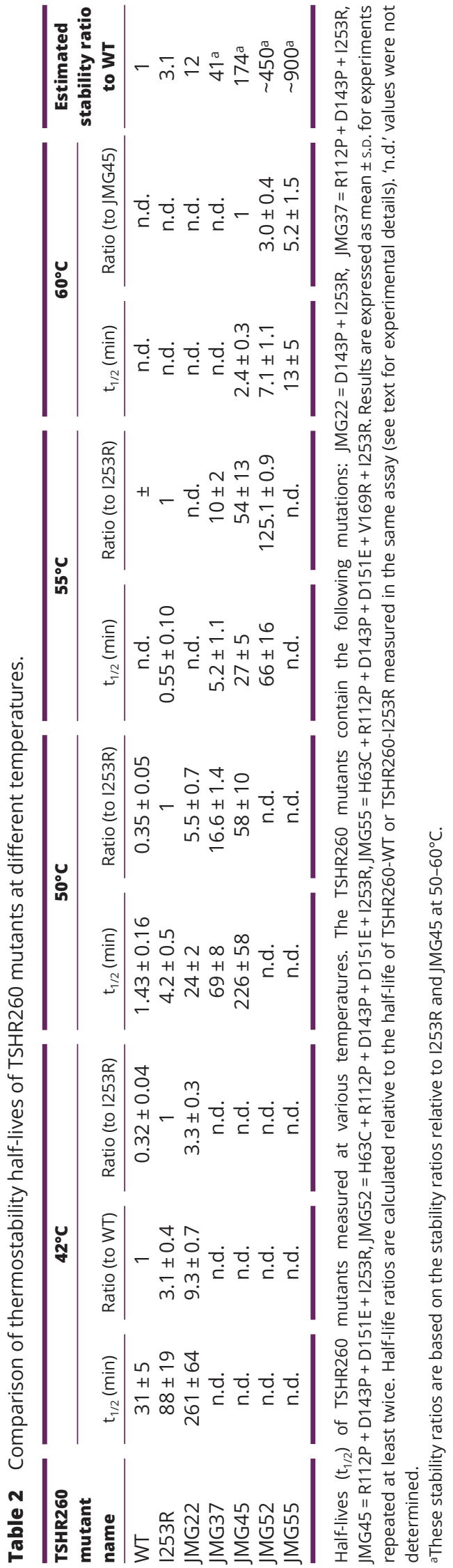

https://jme.bioscientifica.com range of different concentrations of porcine TSH, M22 ${ }^{\mathrm{TM}}$ Fab or K1-18 ${ }^{\mathrm{TM}}$ IgG (Fig. 3). The stimulating effects of porcine TSH and K1-18 ${ }^{\mathrm{TM}}$ on WT TSHR and TSHR-JMG55 $5^{\mathrm{TM}}$ were similar with EC50s of $0.6-0.8 \mathrm{ng} / \mathrm{mL}$, while there was just a small decrease in the stimulation by $\mathrm{M} 22^{\mathrm{TM}}$ from an EC50 of $1.47 \pm 0.09 \mathrm{ng} / \mathrm{mL}$ for WT TSHR to $3.0 \pm 0.3 \mathrm{ng} / \mathrm{mL}$ for TSHR-JMG55 ${ }^{\mathrm{TM}}$ (Fig. 3).

\section{Expression and purification}

Ligand-free TSHR260-JMG55TM remained stable through three rounds of purification, deglycosylation and a further two purification steps and retained activity in the TSHR260 binding assay (Supplementary Table 1, see section on supplementary data given at the end of this article). TSHR260-JMG55 ${ }^{\mathrm{TM}}$ lost $2 \mathrm{kDa}$ of glycans after deglycosylation as observed by SDS-PAGE $12 \%$ acrylamide gel (Supplementary Fig. 1).

\section{Crystal structure}

The crystal structure of TSHR260-JMG55'TM was solved to $2.83 \AA$ resolution. The statistics for data collection and refinement are in Supplementary Table 2. TSHR260JMG55TM crystallised in the space group $\mathrm{P}_{2} 22$, which consists of 12 asymmetric units in a hexagonal unit cell $(\mathrm{a}=\mathrm{b}=144.1 \AA, \mathrm{c}=135.6 \AA)$ and has $63.9 \%$ solvent content. There are two TSHR260-JMG55TM molecules in the asymmetric unit, defined as TSHR-A and TSHR-B. TSHR-A and TSHR-B structures are almost identical (RMSD of $0.42 \AA$ between residues 30-256) except for some differences in the N-termini, therefore detailed descriptions of the TSHR260JMG55TM structure are based on TSHR-A, with TSHR-B described only where it differs from TSHR-A. Electron density is observed for residues $24-260$ which allowed a greater number of residues to be modelled compared to residues 30-257 for the M22 ${ }^{\mathrm{TM}}$-TSHR260 complex (TSHR-M22, PDB code 3G04, Sanders et al. 2007) and residues 24-257 for the K1-70TM-TSHR260 complex (TSHR-K1-70, PDB code 2XWT, Sanders et al. 2011). However, residues 22 and 23 were not visible in the electron density map of TSHR260-JMG55 and the side chains of nine residues (TSHR-A: Glu30, Arg65 and Met140; TSHR-B: Cys24, Arg65, Met140, Lys250, Trp258 and Leu260) could not be unambiguously modelled. These residues may have static or dynamic disorder, which result in dispersed electron density. Due to the $2.83 \AA$ resolution of the solved TSHR260-JMG55TM structure, it was not possible to identify any water molecules in the structure unlike in the higher resolution structures of TSHR-M22 and TSHR-K1-70. 
A

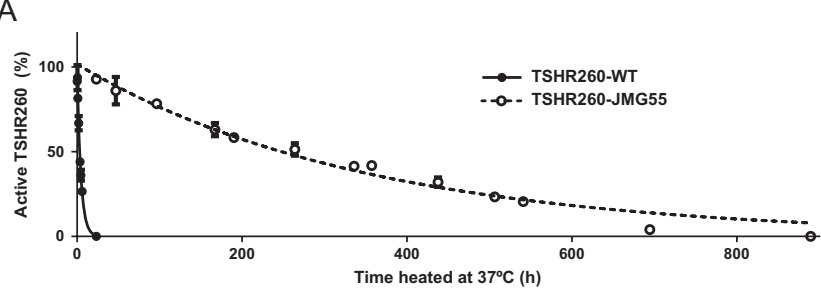

B
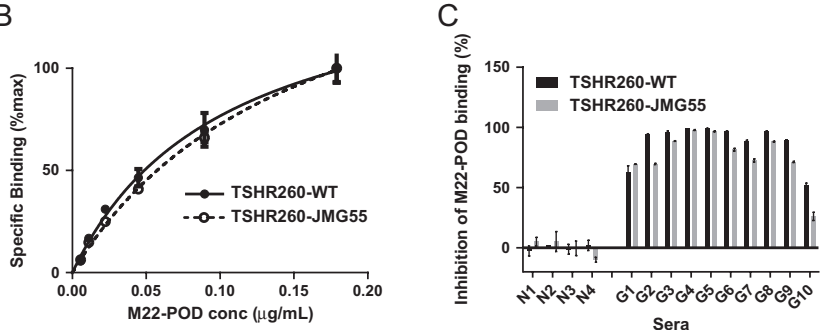

Figure 2

(A) Thermostability assay of TSHR260-WT compared to TSHR260-JMG55TM at $37^{\circ} \mathrm{C}$. (B) Binding affinity of TSHR260-WT $\left(\mathrm{K}_{\mathrm{d}}=0.10 \pm 0.04 \mu \mathrm{g} / \mathrm{mL}, n=3\right)$ and TSHR260-JMG55TM $\left(\mathrm{K}_{d}=0.16 \pm 0.03 \mu \mathrm{g} / \mathrm{mL}, n=3\right)$ for M22-POD. Representative data from experiment repeated three times. (C) Inhibition of M22-POD binding to TSHR260 (black) and TSHR260-JMG55TM (grey) by healthy blood donor sera (N1-4) and Graves' patient sera (G1-10). Standard deviations for data points measured in duplicate are plotted. Reproduced with permission of the copyright holder, RSR Ltd.

The structure of TSHR260-JMG55TM is very similar to the structures of the WT TSHR260 from TSHR-M22 and TSHR-K1-70 (Fig. 4 and Supplementary Fig. 2). As in previous TSHR260 structures, TSHR260-JMG55 ${ }^{\mathrm{TM}}$ has an N-terminal cap (N-cap) and ten LRRs. Each LRR consists of a parallel $\beta$-strand on its concave surface while the $\mathrm{N}$-cap has an additional $\beta$-strand antiparallel to the $\beta$-strand of the first repeat. Altogether, the eleven $\beta$-strands form a $\beta$-sheet on the concave surface of the structure. The arrangement of disulphide bonds in the $\mathrm{N}$-cap is slightly different between the two TSHR260-JMG55 ${ }^{\mathrm{TM}}$ molecules. There are two disulphide bonds (Cys24-Cys29 and Cys31Cys41) in TSHR-A as was observed for TSHR-K1-70 and one disulphide bond (Cys31-Cys41) in TSHR-B, as observed in TSHR-M22. Additionally Cys29 of TSHR-B appears to form an intermolecular disulphide bond with Cys29 of a symmetry-related TSHR-B molecule (Supplementary Fig. 3).

A structural superimposition of the structures of the TSHR-A, TSHR-M22 and TSHR-K1-70 shows that all the structures are very similar apart from the N-termini (Fig. 4A, B and Supplementary Fig. 3A). The structural superimposition of the full structures (residues 24-260 for TSHR-A; 24-257 for TSHR-K1-70; and 30-257 for TSHR-M22) shows RMSDs of $1.46 \AA$ for TSHR-A superimposed to TSHR-K1-70 and $0.75 \AA$ for TSHR-A superimposed to TSHR-M22 (Supplementary Table 3). The RMSD values for TSHR-M22 are lower than that for
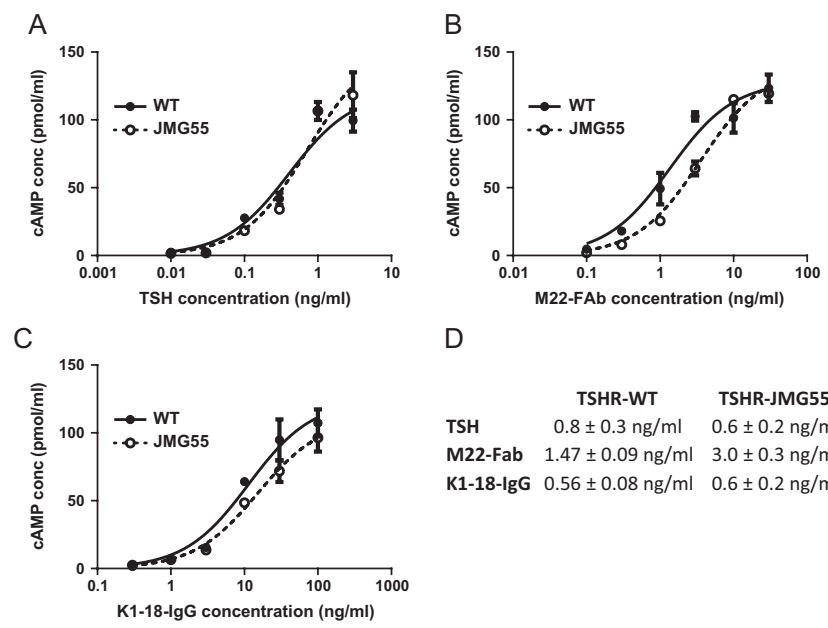

D

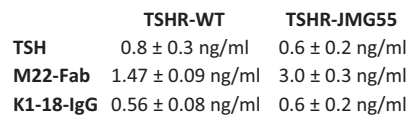

Figure 3

Stimulation of full-length TSHR-WT (solid line, filled circles) and TSHRJMG55TM (dashed line, open circles) stably expressed in Flp-In CHO cells to produce cAMP using (A) porcine TSH, (B) M22 ${ }^{\mathrm{TM}}$-Fab and (C) K1-18 ${ }^{\mathrm{TM}}$-IgG. (D) Half-maximal effective concentrations (EC50) for the different stimulation experiments. Representative data are shown for experiments repeated at least twice. EC50 results are calculated using non-linear regression fitting a stimulation dose-response curve using GraphPad Prism (GraphPad Software Inc. 2015). Standard deviations for data points measured in triplicate are plotted. Reproduced with permission of the copyright holder, RSR Ltd.

TSHR-K1-70 due to the solved structure of TSHR-M22 lacking the $\mathrm{N}$-terminus that shows more variation between the structures than the LRR domains. When residues 30-256 for all four structures, that is excluding the variable N-terminal and the C-terminal residues missing from the TSHR-K1-70 and TSHR-M22 structures were superimposed, the RMSD values were low, 0.60 $0.71 \AA$ (Supplementary Table 3).

In the extracellular domain of the TSHR there are six asparagines that conform to the consensus sequence for N-linked glycosylation (Asn-Xxx-Ser/Thr, where $\mathrm{Xxx}$ is any amino acid except proline), Asn77, Asn99, Asn113, Asn177, Asn198, Asn302 (Núñez Miguel et al. 2017), and previous studies have indicated that all six glycosylation sites are glycosylated (Tanaka et al. 1998, Sanders et al. 2007). All five of the glycosylation sites that are within the TSHR260 domain have observed glycans attached in the TSHR-M22 structure (Sanders et al. 2007) and four of the sites (Asn77, Asn99, Asn177 and Asn198) have glycans observed in the TSHR-K1-70 structure (Sanders et al. 2011). In TSHR260-JMG55 TM, three glycans are observed attached to Asn99, Asn113 and Asn198 of TSHR-A while only two are observed attached to Asn99 and Asn198 of TSHR-B. The differences in observed glycans are most likely due to either disorder in the structure or differences in the partial deglycosylation of TSHR260 before crystallisation. 
A

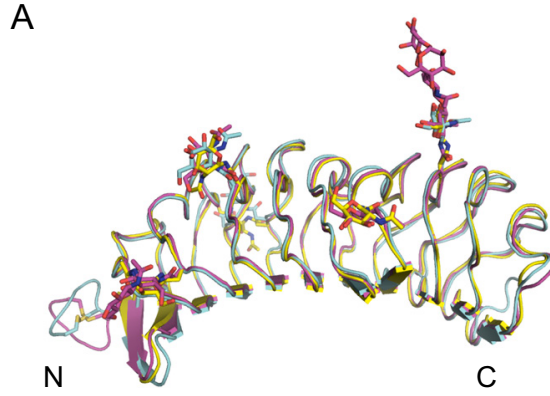

C

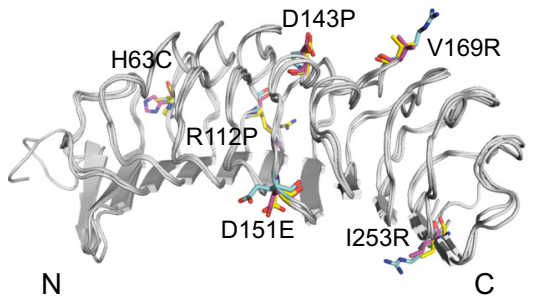

B

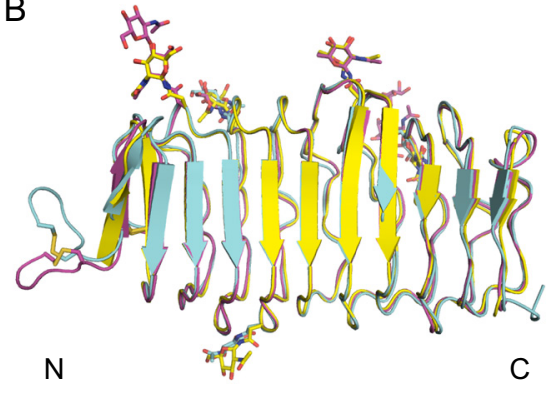

C

D V169R

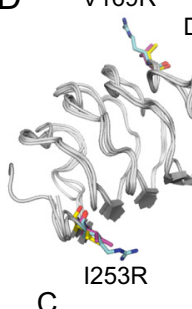

C

Figure 4

Structural superimposition of the structures of TSHR-A (cyan), TSHR-K1-70 (magenta) and TSHRM22 (yellow) observed from (A) the edge of the $L R D$ and $(B)$ rotated $90^{\circ}$ to show the concave surface of the LRD. Structures are in cartoon representation with the disulphide bonds and glycosylation sites in stick representation with oxygen in red, nitrogen blue and sulphur yellow. (C) and (D) Two views rotated $180^{\circ}$ of the structural alignment of TSHR structures with mutated residues, H63C, R112P, D143P, D151E, V169R and I253R, in stick representation coloured by atom (oxygen is red, nitrogen is blue) with the carbon atoms coloured by molecule (TSHR-A is cyan, TSHR-K1-70 is magenta and TSHR-M22 is yellow). Reproduced with permission of the copyright holder, RSR Ltd.

The two TSHR260-JMG55TM molecules in the asymmetric unit, TSHR-A and TSHR-B, interact at their N-termini to form a U-shape (Fig. 5 and Supplementary Fig. 2), which most likely is a crystallographic artefact as this arrangement obscures the M22 ${ }^{\mathrm{TM}}$ binding site on the concave surface of TSHR260, while in our experiments TSHR260-JMG55 ${ }^{\mathrm{TM}}$ preparations bind M22 ${ }^{\mathrm{TM}}$. The asymmetric unit then makes crystal-packing interactions with five other asymmetric units (Fig. 5). The two most interesting crystal-packing interfaces are first at the $\mathrm{N}$-terminus, where there is an intermolecular disulphide bond between two TSHR-B molecules (Supplementary Fig. 3D), and secondly involve LRR2, LRR3 and LRR4 where a zinc ion coordinates interactions between four histidine residues.

\section{Discussion}

TSHR260-JMG55 $5^{\mathrm{TM}}$ produced in this study had similar characteristics to the WT TSHR260 in binding assays and inhibition assays with porcine TSH, human TSHR mAbs $\mathrm{M} 22^{\mathrm{TM}}, \mathrm{K} 1-70^{\mathrm{TM}}$ and $\mathrm{K} 1-18^{\mathrm{TM}}$ and TRAb-positive patient sera indicating that the mutations have not affected the binding properties of the receptor. Furthermore, the ability of various ligands to stimulate production of cyclic AMP in CHO cells expressing full-length TSHR-JMG55TM was similar to CHO cells expressing WT TSHR. Therefore, TSHR260-JMG55 $5^{\mathrm{TM}}$ is suitable for detecting TRAbs and aiding our understanding of TSHR structure and function.

TSHR mutations were introduced on a template of the TSHR260 fragment that had been crystallised previously in complex with M22TM and with K1-70TM (Sanders et al.
2007, 2011). This study design allowed direct comparison of the ligand-free stabilised TSHR LRD fragment to the autoantibody-bound TSHR260.

Previously, truncated forms of the TSHR have been expressed in CHO cells, with TSHR-261 expressing more efficiently than the larger TSHR-289 and both fragments demonstrating TSHR antibody binding (Chazenbalk et al.

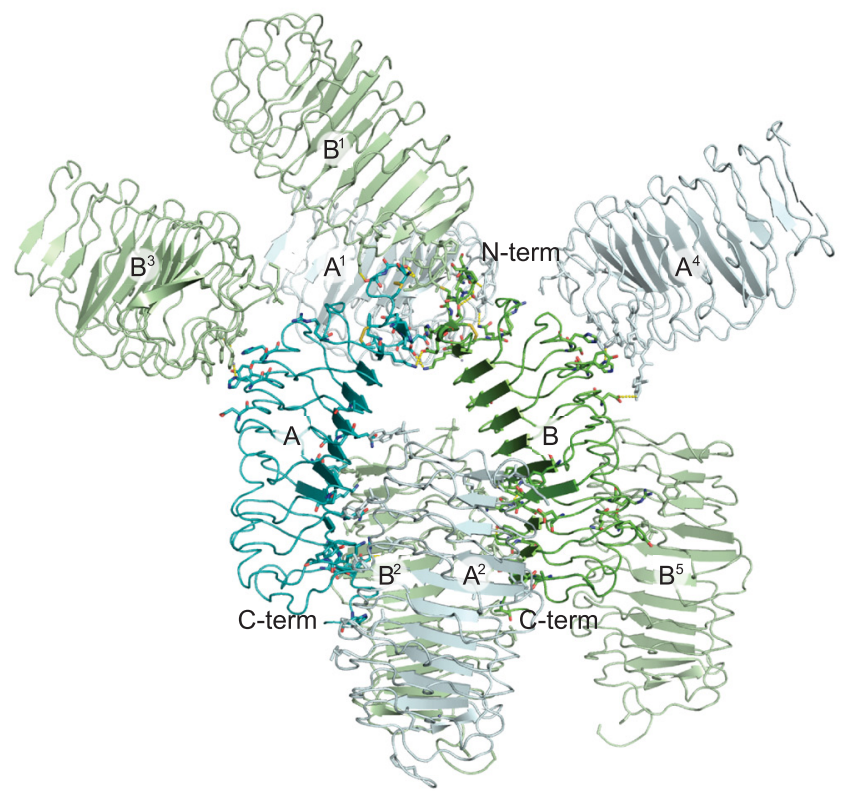

Figure 5

Crystal contacts between TSHR260-JMG55TM molecules. Asymmetric unit of TSHR260-JMG55TM consisting of TSHR-A (cyan) and TSHR-B (green) with symmetry-related subunits that make crystal contacts shown in light cyan (TSHR-A) and light green (TSHR-B). Reproduced with permission of the copyright holder, RSR Ltd. 
$1997 a, b)$. In a following study, the more stable TSHR-289 fragment was separated into 'active' and 'inactive' forms by affinity purification using an inactive TSHR-specific mouse mAb (3BD10) (Chazenbalk et al. 2001). The ability of the 'active' TSHR-289 to recognise Graves' sera was reduced upon heat stressing the receptor and this TSHR inactivation process was reduced by use of chemical chaperones (Chazenbalk et al. 2001).

Unlike WT TSHR260, which we have previously demonstrated is denatured and degraded during expression and purification when produced in the absence of a stabilising mAb (Sanders et al. 2007); ligandfree TSHR260-JMG55TM remained stable and active in the TSHR260 binding assay after five rounds of purification. The stabilising mutations have therefore enabled the complete purification of folded, active, ligand-free TSHR260 for the first time.

\section{Thermostabilising mutations}

The six thermostabilising mutations in TSHR-JMG55 $5^{\mathrm{TM}}$ are located on the surface of TSHR260 rather than in the core of the molecule (Fig. 4C and D) and have side chains clearly visible in the 2Fo-Fc map when contoured at $1 \sigma$. Of the six mutated residues, only Asp151 interacts with M22 ${ }^{\mathrm{TM}}$ and with $\mathrm{K} 1-70^{\mathrm{TM}}$ in their respective complexes with TSHR260 (Sanders et al. 2007, 2011). RMSDs were calculated for the mutated backbone atoms in TSHR260-JMG55 ${ }^{\mathrm{TM}}$ (residues $63,112,143,151,169$ and 253) for the pairs of molecules: TSHR-M22 and TSHR-A; TSHR-K1-70 and TSHR-A; and TSHR-M22 and TSHR-K1-70 (Supplementary Table 4). For most mutated residues, the RMSDs were less than $1 \AA$ indicating that the mutations did not greatly alter the backbone structure of TSHR260. There were more significant changes in the conformations and interactions of the side chains shown in Supplementary Table 4. These changes included formation of new interactions, removal of solvent exposed hydrophobic residues and decreased flexibility, which help explain the increased thermostability of the protein (Pace et al. 1996, 2014).

The presence of His63 in two different conformations in TSHR-M22 and TSHR-K1-70 indicated that this side chain is flexible in WT TSHR260 (Fig. 6A). In TSHR260JMG55TM, the flexible His63 was replaced by the smaller Cys residue, resulting in reduced conformational entropy, which could improve thermostability. Cys63 does not make any intramolecular disulphide bonds, as there are no suitable Cys residues nearby.

Mutation of Arg112 and Asp143 to a torsionally constrained Pro reduces main chain flexibility and could thereby increase thermostability (Fig. 6B and C) as flexible sites are thought to be the first sites of protein unfolding. The $\varphi$ torsion angle of the Arg112 and Asp143 backbones $\left(-67.6^{\circ}\right.$ and $-71.8^{\circ}$ for TSHR-K1-70 and TSHR-M22, respectively) is within the ideal range for Pro $\left(-65^{\circ} \pm 20^{\circ}\right)$; therefore, these mutations were tolerated. Furthermore, substitution of Asp143 by Pro causes residues 144-146 to move $1 \AA$ away from the side chain of residue 143 allowing Lys146 to form a salt bridge with Asp120.

In the TSHR, Asp151 makes interactions with neighbouring residues (Phe153 and Ile152) (Fig. 6D). However, after mutating from Asp to Glu there are no alternative polar interactions with the side chain of Glu151 in the TSHR260-JMG55TM structure that would suggest improved stability. A possible explanation is that the longer side chain of Glu protrudes further from the surface of TSHR260 than Asp, allowing it to make more interactions with the solvent.

The mutation of Val169 to the polar Arg allows the formation of new hydrogen bonds with the glycan attached to Asn198 in TSHR-A (Fig. 6E). Furthermore, removal of the hydrophobic Val residue from the surface and the introduction of the exposed charged side chain of Arg that can make interactions with the solvent could further contribute to the stability of TSHR260.

Also, mutation of Ile253 to Arg allows the formation of new salt bridge interactions with Glu251 and Asp232. Additionally, the exposed charged side chain of Arg can make interactions with the solvent that were not possible for hydrophobic Ile.

\section{$\mathrm{N}$-terminal structure}

The greatest difference in the four TSHR260 structures (TSHR-A and TSHR-B from the TSHR260-JMG55TM structure and TSHR-M22 and TSHR-K1-70) is residues 22-29 at the N-terminus. Although the secondary structures of the N-cap of the TSHR260 are similar for the four structures with residues 31-34 forming a $\beta$-strand, the structure of the first six residues (24-29) is a loop with a highly variable structure across the three molecules showing these residues, TSHR-A, TSHR-B and TSHR-K1-70 (Supplementary Fig. 3A). Even the two molecules in the asymmetric unit of the crystal structure of TSHR260JMG55 ${ }^{\mathrm{TM}}$ show different structures of their $\mathrm{N}$-caps. These differences are partly due to disorder of the region around residues 22-29 which lacked clear electron density in any of the crystal structures (Sander et al. 2007, 2011) and even where the residues have been modelled these were at the limits of the resolution of the structures (Supplementary 
A

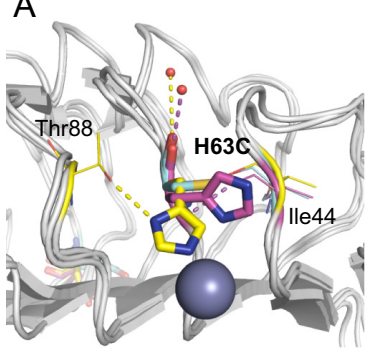

B
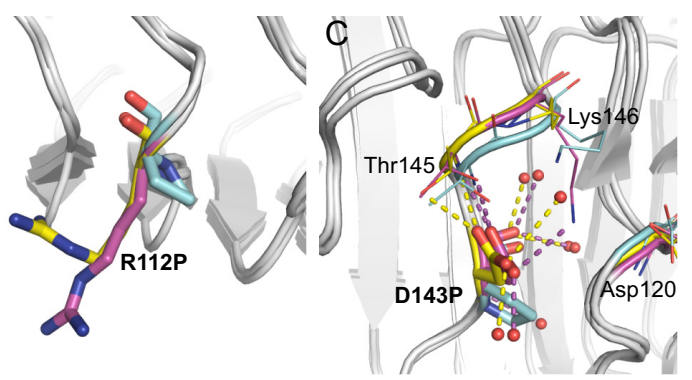

E
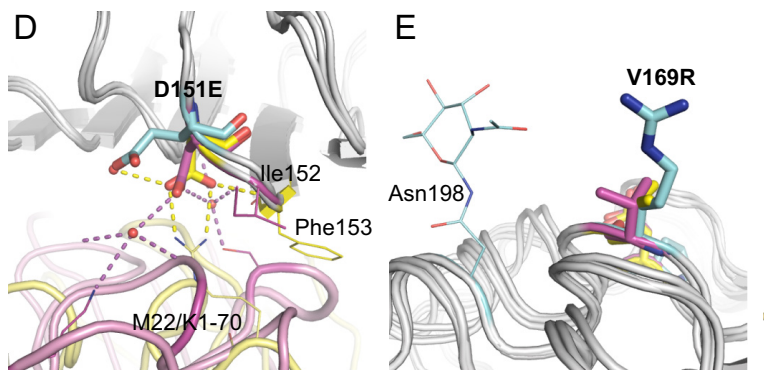

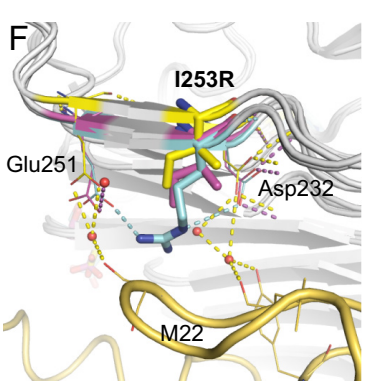

Figure 6

Comparison of the mutated residues: (A) H63C (B) R112P, (C) D143P, (D) D151E, (E) V169R and (F) I253R, in TSHR-A, TSHR-M22 and TSHR-K1-70. Mutated residues are shown in stick representation with other interacting residues in line representation. Residues are coloured by atom (oxygen is red, nitrogen blue, sulphur yellow) with the carbon atoms and non-covalent bonds (dashed lines) coloured by molecule (TSHR-A is cyan, TSHR-K1-70 is magenta and TSHR-M22 is yellow). $\mathrm{Zn}^{2+}$ is shown as a sphere. Reproduced with permission of the copyright holder, RSR Ltd.
Fig. 3B and C). The N-terminal intermolecular interactions observed in the TSHR260-JMG55TM crystal structure occurred in a similar location to those of a modelled TSHR by Chen et al. (2015). However, the relative positions of the $\mathrm{N}$-terminal intermolecular interactions in the modelled TSHRs (which formed a trimer) and the interactions seen in the TSHR260-JMG55 ${ }^{\mathrm{TM}}$ crystal structure differed. Consequently, it is not possible from our data to assess the importance of these N-terminal interactions in the crystal structure. It is likely they are a result of crystal packing.

The disulphide bond Cys31-Cys41, which has been found to be important for the structure and function of the TSHR (Chen et al. 2001), is present in all four structures (Supplementary Fig. 3A). In addition, TSHR-A and TSHR-K1-70 show a disulphide bond between Cys24 and Cys29, whereas in TSHR-B, Cys29 forms an intermolecular disulphide bond with a Cys29 residue of a symmetry-related TSHR-B molecule (molecule $\mathrm{B}^{1}$ in Fig. 5 and Supplementary Fig. 3D). Examination of the difference map (Fo-Fc) reveals some positive unmodelled electron density between Cys29 and Cys24 of TSHR-B (green mesh in Supplementary Fig. 3C), suggesting that there could be a partially occupied disulphide bond between these residues and Cys29 could form a mixture of disulphide bonds in the structure.

\section{Comparison of the ligand-free and autoantibody- bound TSHR260 structures}

The backbones of ligand-free and autoantibody (M22 or $\mathrm{K} 1-70^{\mathrm{TM}}$ ) bound TSHR260 structures are essentially unchanged (Fig. 4 and Supplementary Table 3) except
(C) 2019 Society for Endocrinology Published by Bioscientifica Ltd. Printed in Great Britain for the disordered N-termini. However, many of the side chains of TSHR260 residues interact with the antibodies in the structures TSHR-M22 and TSHR-K1-70. Some of these residues do not change conformation when the antibodies bind to TSHR260, while others rearrange their non-covalent interactions to form new non-covalent interactions with the antibodies (Supplementary Fig. 4).

In addition, some flexible side chains with poorly resolved electron density in TSHR-A (Glu35, Asp36, Arg38, Lys42, Lys57, Arg80, Lys129, Ile155, Lys183, Lys209 and Arg255) have clearer electron density in TSHR-M22 and/ or TSHR-K1-70 structures suggesting they are stabilised by their interactions with M22 ${ }^{\mathrm{TM}}$ and/or K1-70 (Supplementary Fig. 4). Mutation of these residues to amino acids with smaller side chains may further improve the thermostability of ligand-free TSHR260, although binding of $\mathrm{M} 22^{\mathrm{TM}}$ and $\mathrm{K} 1-70^{\mathrm{TM}}$ to the receptor may be affected. A more detailed description of the comparison between ligand-free and -bound TSHR260 can be found in the supplementary information.

\section{Oligomerisation}

It has been proposed that glycoprotein hormone receptors form oligomers (Fan \& Hendrickson 2005, Jiang et al. 2012, 2014, Chen et al. 2015). However, purified TSHR260 in complex with M22 ${ }^{\mathrm{TM}}-\mathrm{Fab}$ or K1-70TM-Fab and the ligandfree TSHR260-JMG55 ${ }^{\mathrm{TM}}$ ran as monomers on size exclusion chromatography. Furthermore, in the TSHR-K1-70 crystal structure, no TSHR intermolecular contacts are observed and this is consistent with the monomer seen on size exclusion chromatography (Sanders et al. 2011). In the 
TSHR-M22 structure, there are crystal-packing interactions on the convex surface between residues in LRR2 (His70), LRR3 (Gln91, Glu93 and His95) and LRR4 (Asp118 and Asp120) of two symmetry-related TSHR260 molecules coordinated by three zinc ions. These interactions are similar to the crystal-packing interactions on the convex surface of TSHR260-JMG55 (Fig. 5), which have a zinc ion coordinating interactions between LRR2 (His70), LRR3 (His95) and LRR4 (Asp120) of two TSHR260 molecules (molecules $\mathrm{A}$ and $\mathrm{B}^{3}$ or $\mathrm{A}^{4}$ and $\mathrm{B}$ in Fig. 5). The residues, His70, His95 and Asp120, are located in an area that has been previously predicted as a possible oligomerisation site (Núñez Miguel et al. 2017). However, the relative orientations of the molecules in each dimer are different. Therefore, it is likely that these intermolecular contacts are a result of crystal packing.

The crystal structure of TSHR260-JMG55 ${ }^{\mathrm{TM}}$ provided rational explanations for the thermostabilising effect of the six mutations used in this study. This study has shown that an alternative strategy for selecting mutations aimed at thermostabilising proteins could involve examining available crystal structures with the view of finding the flexible residues that would be good candidates for mutation. These residues would not be in the active site, would be in multiple conformations or would have poorly resolved electron density. Mutation of flexible residues to smaller residues, proline or polar residues may increase the thermostability of the protein.

The crystal structure of TSHR260 in its apo form has shown remarkable similarity to the TSHR260 structures bound to $\mathrm{M} 22^{\mathrm{TM}}$ or $\mathrm{K} 1-70^{\mathrm{TM}}$, with very small RMSDs for the backbones and only a few changes in the more flexible $\mathrm{N}$ - and $\mathrm{C}$-termini and some of the side chains that interact with $\mathrm{M} 22^{\mathrm{TM}}$ and/or K1-70 ${ }^{\mathrm{TM}}$. It has been previously suggested that due to steric hindrance of autoantibody binding to the TSHR LRD (Chazenbalk et al. 2002, Mizutori et al. 2009) high affinity binding between autoantibodies and the TSHR must be associated with torsion in the hinge region (Rapoport \& McLachlan 2007). Our study shows that there is very little change in the TSHR260 structure on the binding of either stimulating $\mathrm{M} 22^{\mathrm{TM}}$ or blocking $\mathrm{K} 1-70^{\mathrm{TM}}$, therefore it is difficult to infer the wider consequences of autoantibody binding on movement of other parts of the TSHR relative to each other. Consequently, more information is needed to improve our understanding of how initial binding of ligands such as $\mathrm{M} 22^{\mathrm{TM}}$ cause TSHR activation. Also, future studies using stabilised TSHRs in analyses of signalling pathways involved in activation of the TSHR by different ligands may be helpful.
Overall, thermostable preparations of TSHR260 described in this study are promising tools for developing new methods of TRAb detection including agglutination PCR (Tsai et al. 2016, 2018). Also they should be useful in therapeutic interventions such as specific immunoadsorption of TSHR autoantibodies.

\section{Supplementary data}

This is linked to the online version of the paper at https://doi.org/10.1530/ JME-18-0213.

\section{Declaration of interest}

RSR Ltd. is a developer of in vitro medical diagnostics including kits for measuring thyroid autoantibodies. All authors are employees of RSR Ltd. A patent application based on these results has been filed.

\section{Funding}

The work was funded by RSR Ltd.

\section{References}

Accelrys Software Inc. 2012 Discovery Studio 3.5. San Diego, CA, USA: Accelrys Software Inc.

Battye TGG, Kontogiannis L, Johnson O, Powell HR \& Leslie AGW 2011 iMOSFLM: a new graphical interface for diffraction-image processing with MOSFLM. Acta Crystallographica. Section D, Biological Crystallography 67 271-281. (https://doi.org/10.1107/S09074444910048675)

Chazenbalk GD, Jaume JC, McLachlan SM \& Rapoport B 1997a Engineering the human thyrotropin receptor ectodomain from a non-secreted form to a secreted, highly immunoreactive glycoprotein that neutralizes autoantibodies in Graves' patients' sera. Journal of Biological Chemistry 272 18959-18965. (https://doi.org/10.1074/ jbc.272.30.18959)

Chazenbalk GD, Tanaka K, Nagayama Y, Kakinuma A, Jaume JC, McLachlan SM \& Rapoport B $1997 b$ Evidence that the thyrotropin receptor ectodomain contains not one, but two, cleavage sites. Endocrinology 138 2893-2899. (https://doi.org/10.1210/ endo.138.7.5259)

Chazenbalk GD, McLachalan SM, Pichurin P, Yan XM \& Rapoport B 2001 A prion-like shift between two conformational forms of a recombinant thyrotropin receptor A-subunit module: purification and stabilization using chemical chaperones of the form reactive with Graves' autoantibodies. Journal of Clinical Endocrinology and Metabolism 86 1287-1293. (https://doi.org/10.1210/jcem.86.3.7363)

Chazenbalk GD, Pichurin P, Chen C-R, Latrofa F, Johnstone AP, McLachlan SM \& Rapoport B 2002 Thyroid-stimulating autoantibodies in Graves' disease preferentially recognise the free A subunit, not the thyrotropin holoreceptor. Journal of Clinical Investigation 110 209-217. (https://doi.org/10.1172/JC1200215745)

Chen CR, Tanaka K, Chazenbalk GD, McLachlan SM \& Rapoport B 2001 A full biological response to Autoantibodies in Graves' disease requires a disulfide-bonded Loop in the thyrotropin receptor $\mathrm{N}$ terminus homologous to a laminin epidermal growth factor-like domain. Journal of Biological Chemistry 276 14767-14772. (https:// doi.org/10.1074/jbc.M008001200)

Chen CR, Hubbard PA, Salazar LM, McLachlan SM, Murali R \& Rapoport B 2015 Crystal structure of a TSH receptor monoclonal 
antibody: insight into Graves' disease pathogenesis. Molecular Endocrinology 29 99-107. (https://doi.org/10.1210/me.2014-1257)

de Bernard S, Misrahi M, Huet JC, Beau I, Desroches A, Loosfelt H, Pichon C, Pernollet JC \& Milgrom E 1999 Sequential cleavage and excision of a segment of the thyrotropin receptor ectodomain. Journal of Biological Chemistry 274 101-107. (https://doi.org/10.1074/jbc.274.1.101)

Emsley P, Lohkamp B, Scott WG \& Cowtan K 2010 Features and development of coot. Acta Crystallographica. Section D, Biological Crystallography 66 486-501. (https://doi.org/10.1107/ S0907444910007493)

Evans PR \& Murshudov GN 2013 How good are my data and what is the resolution? Acta Crystallographica. Section D, Biological Crystallography 69 1204-1214. (https://doi.org/10.1107/ S0907444913000061)

Evans M, Sanders J, Tagami T, Sanders P, Young S, Roberts E, Wilmot J, Hu X, Kabelis K, Clark J, et al. 2010 Monoclonal autoantibodies to the TSH receptor, one with stimulating activity and one with blocking activity, obtained from the same blood sample: monoclonal autoantibodies to the TSH receptor. Clinical Endocrinology $\mathbf{7 3}$ 404-412. (https://doi.org/10.1111/j.1365-2265.2010.03831.x)

Fan QR \& Hendrickson WA 2005 Structure of human follicle-stimulating hormone in complex with its receptor. Nature 433 269-277. (https:// doi.org/10.1038/nature03206)

Furmaniak J, Sanders J \& Rees Smith B 2013 Blocking type TSH receptor antibodies. Auto-Immunity Highlights 4 11-26. (https://doi. org/10.1007/s13317-012-0028-1)

Furmaniak J, Sanders J, Miguel RN \& Rees Smith B 2015 Mechanisms of action of TSHR autoantibodies. Hormone and Metabolic Research $\mathbf{4 7}$ 735-752. (https://doi.org/10.1055/s-0035-1559648)

GraphPad Software Inc. 2015 GraphPad Prism Version 6.07 for Windows. La Jolla, CA, USA: GraphPad Software Inc.

Hsu SY, Kudo M, Chen T, Nakabayashi K, Bhalla A, van der Spek PJ, van Duin M \& Hsueh AJW 2000 The three subfamilies of leucine-rich repeat-containing $\mathrm{G}$ protein-coupled receptors (LGR): identification of LGR6 and LGR7 and the signaling mechanism for LGR7. Molecular Endocrinology 14 1257-1271. (https://doi.org/10.1210/ mend.14.8.0510)

Jiang X, Liu H, Chen X, Chen P-H, Fischer D, Sriraman V, Yu HN, Arkinstall S \& He X 2012 Structure of follicle-stimulating hormone in complex with the entire ectodomain of its receptor. PNAS $\mathbf{1 0 9}$ 12491-12496. (https://doi.org/10.1073/pnas.1206643109)

Jiang X, Fischer D, Chen X, McKenna SD, Liu H, Sriraman V, Yu HN, Goutopoulos A, Arkinstall S \& He X 2014 Evidence for folliclestimulating hormone receptor as a functional trimer. Journal of Biological Chemistry 289 14273-14282. (https://doi.org/10.1074/jbc. M114.549592)

Kleinau G, Worth CL, Kreuchwig A, Biebermann PM, Scheerer P, Krause G 2017 Structural-functional features of the thyrotropin receptor: a class A G-protein-coupled receptor at work. Frontiers in Endocrinology 886 (https://doi.org/10.3389/fendo.2017.0086)

Lebon G, Bennett K, Jazayeri A \& Tate CG 2011 Thermostabilisation of an Agonist-Bound Conformation of the Human Adenosine A2A Receptor. Journal of Molecular Biology 409 298-310. (https://doi. org/10.1016/j.jmb.2011.03.075)

Magnani F, Serrano-Vega MJ, Shibata Y, Abdul-Hussein S, Lebon G, Miller-Gallacher J, Singhal A, Strege A, Thomas JA \& Tate CG 2016 Thermostabilisation of membrane proteins for structural studies. Nature Protocols 11 1554-1571. (https://doi.org/10.1038/ nprot.2016.088)

McCoy AJ, Grosse-Kunstleve RW, Adams PD, Winn MD, Storoni LC \& Read RJ 2007 Phaser crystallographic software. Journal of Applied Crystallography 40 658-674. (https://doi.org/10.1107/ S0021889807021206)

McKenzie JM \& Zakarija M 1992 Fetal and neonatal hyperthyroidism and hypothyroidism due to maternal TSH receptor antibodies. Thyroid 2 155-159. (https://doi.org/10.1089/thy.1992.2.155)
Miller JL \& Tate CG 2011 Engineering an ultra-thermostable $\beta 1$ adrenoceptor. Journal of Molecular Biology 413 628-638. (https://doi. org/10.1016/j.jmb.2011.08.057)

Mizutori Y, Chen C-R, Latrofa F, McLachlan SM \& Rapoport B 2009 Evidence that the shed thyrotropin receptor A subunits drive affinity maturation of autoantibodies causing Graves' disease. Endocrinology and Metabolism 94 927-935. (https://doi.org/10.1210/jc.2008-2134)

Murshudov GN, Skubák P, Lebedev AA, Pannu NS, Steiner RA, Nicholls RA, Winn MD, Long F \& Vagin AA 2011 REFMAC5 for the refinement of macromolecular crystal structures. Acta Crystallographica Section D: Biological Crystallography 67 355-367. (https://doi.org/10.1107/S0907444911001314)

Núñez Miguel R, Sanders J, Jeffreys J, Depraetere H, Evans M, Richards T, Blundell TL, Rees Smith B \& Furmaniak J 2004 Analysis of the thyrotropin receptor-thyrotropin interaction by comparative modeling. Thyroid 14 991-1011. (https://doi.org/10.1089/ thy.2004.14.991)

Núñez Miguel R, Sanders J, Furmaniak J \& Rees Smith B 2017 Glycosylation pattern analysis of glycoprotein hormones and their receptors. Journal of Molecular Endocrinology 58 25-41. (https://doi. org/10.1530/JME-16-0169)

Pace CN, Shirley BA, McNutt M \& Gajiwala K 1996 Forces contributing to the conformational stability of proteins. FASEB Journal 10 75-83. (https://doi.org/10.1096/fasebj.10.1.8566551)

Pace CN, Scholtz JM \& Grimsley GR 2014 Forces stabilizing proteins. FEBS Letters $\mathbf{5 8 8}$ 2177-2184. (https://doi.org/10.1016/j. febslet.2014.05.006)

Rapoport B and McLachlan SM 2007 The thyrotropin receptor in Graves' disease. Thyroid 17 911-922. (https://doi.org/10.1089/ thy.2007.0170)

Rapoport B \& McLachlan SM 2016 TSH receptor cleavage into subunits and shedding of the A-subunit; a molecular and clinical perspective. Endocrine Reviews 37 114-134. (https://doi.org/10.1210/er.2015-1098)

Rapoport B, Chazenbalk GD, Jaume JC \& McLachlan SM 1998 The thyrotropin (TSH) receptor: interaction with TSH and autoantibodies. Endocrine Reviews 19 673-716. (https://doi.org/10.1210/ edrv.19.6.0352)

Rapoport B, Aliesky HA, Chen C-R \& McLachlan SM 2015 Evidence that TSH receptor A-Subunit Multimers, not monomers, drive antibody affinity maturation in Graves' disease. Journal of Clinical Endocrinology and Metabolism 100 E871-E875. (https://doi.org/10.1210/jc.2015-1528)

Rees Smith B, McLachlan SM \& Furmaniak J 1988 Autoantibodies to the thyrotropin receptor. Endocrine Reviews 9 106-121. (https://doi. org/10.1210/edrv-9-1-106)

Rees Smith B, Bolton J, Young S, Collyer A, Weeden A, Bradbury J, Weightman D, Perros P, Sanders J \& Furmaniak J 2004 A new assay for thyrotropin receptor autoantibodies. Thyroid 14 830-835. (https://doi.org/10.1089/thy.2004.14.830)

Rees Smith B, Sanders J \& Furmaniak J 2007 TSH receptor antibodies. Thyroid 17 923-938. (https://doi.org/10.1089/thy.2007.0239)

Rees Smith B, Sanders J, Evans M, Tagami T \& Furmaniak J 2009 TSH receptor - autoantibody interactions. Hormone and Metabolic Research 41 448-455. (https://doi.org/10.1055/s-0029-1220913)

Sanders J, Oda Y, Roberts S-A, Maruyama M, Furmaniak J \& Rees Smith B 1997 Understanding the thyrotropin receptor functionstructure relationship. Baillière's Clinical Endocrinology and Metabolism 11 451-479. (https://doi.org/10.1016/S0950-351X(97)80693-3)

Sanders J, Evans M, Premawardhana L, Depraetere H, Jeffreys J, Richards T, Furmaniak J \& Rees Smith B 2003 Human monoclonal thyroid stimulating autoantibody. Lancet 362 126-128. (https://doi. org/10.1016/S0140-6736(03)13866-4)

Sanders J, Bolton J, Sanders P, Jeffreys J, Nakatake N, Richards T, Evans M, Kiddie A, Summerhayes S, Roberts E, et al. 2006 Effects of TSH receptor mutations on binding and biological activity of monoclonal antibodies and TSH. Thyroid 16 1195-1206. (https://doi. org/10.1089/thy.2006.16.1195) 
Sanders J, Chirgadze DY, Sanders P, Baker S, Sullivan A, Bhardwaja A, Bolton J, Reeve M, Nakatake N, Evans M, et al. 2007 Crystal structure of the TSH receptor in complex with a thyroid-stimulating autoantibody. Thyroid 17 395-410. (https://doi.org/10.1089/thy.2007.0034)

Sanders P, Young S, Sanders J, Kabelis K, Baker S, Sullivan A, Evans M, Clark J, Wilmot J, Hu X, et al. 2011 Crystal structure of the

TSH receptor bound to a blocking type TSHR autoantibody. Journal of Molecular Endocrinology 46 81-99. (https://doi.org/10.1530/ JME-10-0127)

Schrödinger, LLC 2015 The PyMOL Molecular Graphics System, Version 1.8. New York City, NY, USA: Schrödinger, LLC.

Serrano-Vega MJ, Magnani F, Shibata Y \& Tate CG 2008 Conformational thermostabilization of the $\beta 1$-adrenergic receptor in a detergentresistant form. PNAS 105 877-882. (https://doi.org/10.1073/ pnas.0711253105)

Tanaka K, Chazenbalk GD, McLachlan SM \& Rapoport B 1998 Thyrotropin receptor cleavage at site 1 does not involve a specific amino acid motif but instead depends on the presence of the unique, 50 amino acid insertion. Journal of Biological Chemistry 273 1959-1963. (https://doi.org/10.1074/jbc.273.4.1959)

Tsai C, Robinson PV, Spencer CA \& Bertozzi CR 2016 Ultrasensitive antibody detection by agglutination-PCR (ADAP). ACS Central Science 2 139-147. (https://doi.org/10.1021/ acscentsci.5b00340)

Tsai C, Robinson PV, Cortez PdJ, Elma MLB, Seftel D, Pourmandi N, Pandori MW \& Bertozzi CR 2018 Antibody detection by agglutination-PCR (ADAP) enables early diagnosis of HIV infection by oral fluid analysis. PNAS 115 1250-1255. (https://doi. org/10.1073/pnas.1711004115)

Winn MD, Ballard CC, Cowtan KD, Dodson EJ, Emsley P, Evans PR, Keegan RM, Krissinel EB, Leslie AGW, McCoy A et al. 2011 Overview of the CCP4 suite and current developments. Acta Crystallographica D67 235-242. (https://doi.org/10.1107/ S0907444910045749)

Received in final form 6December2018

Accepted 28January2019

Accepted Preprint published online 28January2019
(C) 2019 Society for Endocrinology Published by Bioscientifica Ltd. Printed in Great Britain 